\title{
Design of Wi-Fi based IoT Sensor Node with Multiple Sensor Types
}

\author{
C.M. Shashika Madushan \\ Department of Electrical and \\ Electronic Technology \\ University of Vocational \\ Technology, Ratmalana, Sri Lanka
}

\author{
M.W.P. Maduranga \\ Department of Computer \\ Engineering \\ General Sir John Kotelawala \\ Defence University, Ratmalana, Sri \\ Lanka
}

\author{
P.I. Ishan Madushanka \\ Department of Electrical and \\ Electronic Technology \\ University of Vocational \\ Technology, Ratmalana, Sri Lanka
}

\begin{abstract}
This paper presents a design and implementation of IoT sensor nodes that can be used for many applications such as smart agriculture, animal detection, building health monitoring, etc. Sensor nodes are designed on a low-cost WiFi-based ESP8266 platform which enables IEEE 802.11n protocol. In this work, we have used two NodeMCU modules (ESP8266) which are easily programmable. NodeMCU which acts itself as a sensor node can be used as Access Point (AP) or as a station (STA). We used one node as an AP and another as an STA. We implemented sensor nodes with DHT 11 sensors which can measure temperature and humidity in the atmosphere. We have done a completed design for the sensor node including Printed Circuit Boards (PCB), waterproofed Enclosures, displays, etc. All the data from sensor nodes can be monitor and record using a remote IoT cloud. For testing, we collected temperature and humidity data using two sensor nodes and analyzed them using MATLAB.
\end{abstract}

\section{Keywords}

IoT, ESP8266, Sensor Networks, Embedded systems, DHT11

\section{INTRODUCTION}

As computing and wireless communications technologies [1] advance and exciting new Internet of Things (IoT)[2] networks emerge, more and more cities and college campuses are becoming smarter and implementing these technologies to exchange data means to do. The goals of "smart" are to simplify the management process, manage real-time access control, and monitor the security of the environment. For this design, we decided to design our IoT sensor node to monitor environmental conditions such as temperature and humidity. First, let's consider what temperature and humidity [3][4].

Absolute Humidity(AH):- Absolute humidity can be defined as "The amount of mass of water vapor present in a unit volume". Such as Equation 1 shown below.

$$
A H=\frac{\text { Mass of water vapor }\left(m_{\mathrm{H}_{2} \mathrm{O}}\right)}{\text { Total volume }(\text { Air }+ \text { Water vapor })}
$$

Relative Humidity(RH):- Relative humidity is the ratio of the partial pressure of the vapor in a gaseous mixture of air and water to the saturated vapor pressure of water at a given temperature. Such as Equation 2 shown below.

$R H=\frac{P_{\mathrm{H}_{2} \mathrm{O}}}{P_{\text {Saturated water vapor }}}$
Temperature: - Temperature is a feeling of warmth or coolness and the manifestation of thermal energy. It can be measured by thermometers. When we consider atmosphere temperature at different levels of the earth's that depends on many factors, Such as Solar radiation, humidity, and altitude

\section{LITERATURE REVIEW}

The main function of a weather station is to provide weather information for forecasting under different environmental conditions. There are many types of weather stations in the world, such as personal weather stations and home weather stations. The weather information provided by these weather stations is temperature, humidity, atmospheric pressure, wind speed, etc. These measurements can be divided into two parts, such as Manual observations measurements and automated measurements. Manual observations are taken at least once daily, while automated measurements are taken at least once an hour.

At present, various agencies and individuals around the world have developed various levels of equipment to obtain this weather information. The disadvantage of that equipment is high cost, not portable, Not error identification method, etc. we tried to avoid those weaknesses and develop device with new features, such as low cost, portability, simplicity, Ergonomic, availability of online access, with error notification system.

\section{DESIGN AND IMPLEMENTATION}

The architecture of the system and it has five main components shown in Figure 1. The main device with display unit, a SD card logger, the Thinger.io webserver (cloudbased data storage and analytics), FTTT web-based Service (Error detection) and Smartphone.

The device utilizes a DHT11 sensor that sensing values about environmental temperature and humidity send to the Thinger.io web server \& store in a data bucket through the "Wi-Fi" connection [5]. If there any situation without Wi-Fi, the system automatically changes from "Wi-Fi Mode" to "SD Mode" then all set of data store in Micro SD memory chip. The device was designed as a compact mobile device with weight $(70 \mathrm{~g})$ and very low power consumption. The device does not use any buttons, display, or indicator ( For the Main device ) to achieve those goals. When the device is powered on, It will continuously measure the temperature and humidity of the environment and send that set of data every 5 minutes to the Thinger.io webserver. Then the user can monitor realtime data by a local computer or smartphone. 
Power consumption: The NodeMCU of the device stay in sleep mode at about 4 minutes and 55 seconds then, it becomes an operational condition (around 5S) to send data to the thinger.io webserver. Therefore this device's power consumption is very low [6].

Error detection: The system includes the method of error detection. If there are any situations such as lose the set of data from DHT11 (ex:- loss connectivity of data wire, VCC, GND between NodeMCU \& DHT11) automatically triggering IFTTT by the endpoint of Thinger.io, as a result of that user can be known about the error by SMS notification [7].

The display unit is most important to monitoring data when Wi-Fi is not available. When we consider the display unit there are two main components included here, the display module and the charging unit. If a user wants to recharge the main device's battery, it needs to be plugged with a "Display unit", and connect with external power $(5.5 \mathrm{~V})$ with a standard USB charger to a USB 2 micro-b charging port.

Finally, it can be exported all data from thinger.io to local computer and analysis data by analyzing software (MATLAB 2017 a)

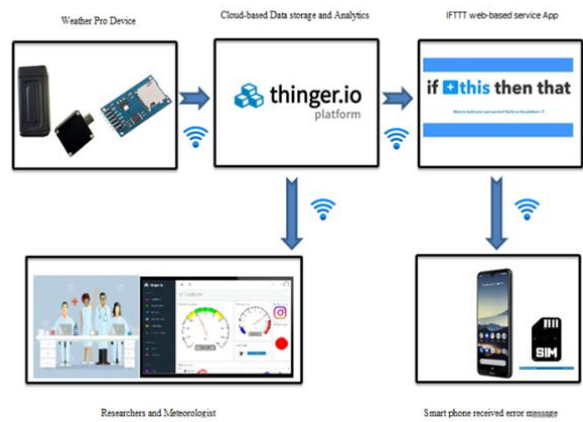

Fig 1 - Architecture of the IoT function

\subsection{Electronic System Design}

The device has two main parts such as (1) Main device (contents:- NodeMCU, DC-DC step-up booster, SD card module, LIPO battery) (2) Display unit (contents:- OLED, charger module). A NodeMCU V3 development board use to design and prototype of the device. The microcontroller (Xtensa LX106) contains a 32-Bit ARM and processor operating at $80 \mathrm{MHz}$. This board (NodeMCU) enables to collecting data by sensor and feed that data to the thinger.io web server through Wi-Fi connection( ESP8266 ) [9][10].

According to electronic design, we used the DHT11 sensor to measure the temperature and humidity of the surroundings and provide calibrated (8-Bit Serial data) digital signal to NodeMCU [11]. The SD card module is connected with the NodeMCU board which helps to store data (read \& writeFAT16 or FAT32 formatted), when there is no "Wi-Fi" [12].

When we considered OLED [13] (128×64 pixels, 0.96 Inches), it has an I2C interface to connect with NodeMCU and it does not need incremental voltage by a booster. But all other devices (NodeMCU, DHT11, SD card module) have a boosted voltage $(5 \mathrm{~V})$ through the DC-DC step-up mini booster module. This step-up booster help converts the DC voltage of the battery (Lithium-ion polymer, $700 \mathrm{mAh}$, and $3.7 \mathrm{~V})$ to $5 \mathrm{~V}(\mathrm{DC})$ ).

When the battery power is low, we can use the display unit with an inbuilt battery charger (TP4056 1A Li-ION battery charger module). This charging module has a mini USB port to connect the external power sources. Electronic system design, as shown in Figure 2.

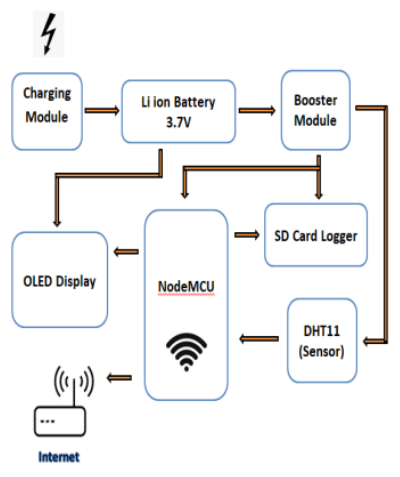

Fig 2 - Electronic System Design

\subsection{Flowchart of the Programming}

First of all the system checked the connection of Wi-Fi to the selected operational mode (Ex: Wi-Fi or SD mode). If there is a Wi-Fi connection, then the Wi-Fi mode is selected more, or else SD mode is selected. Then checked availability of DHT11 sensor reading. If there are sensor readings are available, those readings are sent to the thinger.io web server for monitoring and storing every 5 minutes. Under the delay period, the system goes to sleep mode and repeats the program. If there are no sensor readings are available, created the Endpoint of thinger.io and triggered the IFTTT web service.Then sent error notification to smartphone. Flowchart under wifi mode, as shown in see Fig 3.

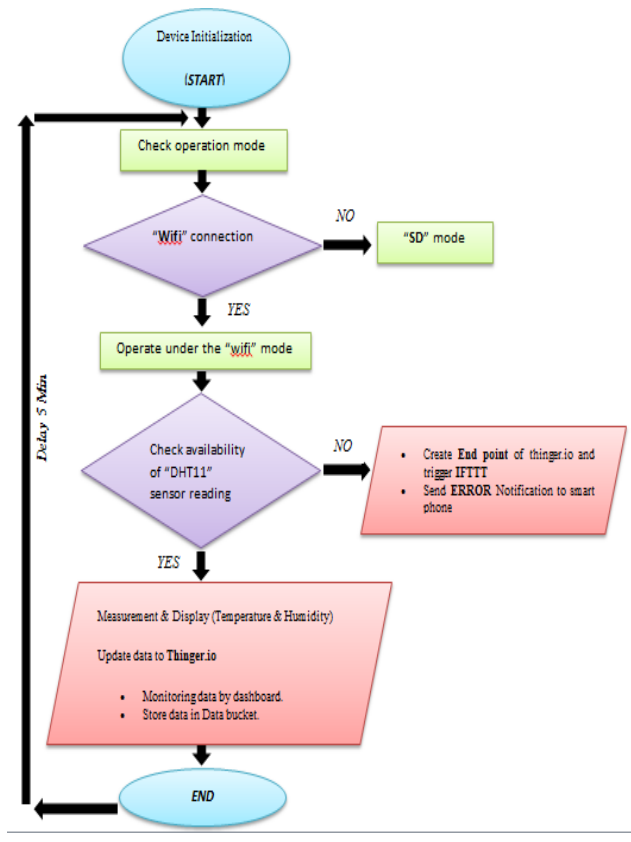

Fig 3 - Flowchart of Firmware design \& code of "With Wi-Fi Mode"

First of all the system checked the connection of Wi-Fi to the selected operational mode (Ex: Wi-Fi or SD mode). If there is no Wi-Fi connection, then SD mode is selected Then checked availability of DHT11 sensor reading. (4) If there are sensor readings are available, those readings are sent to the SD card module and storing per every 5 minutes on the memory chip. Under the delay period, the system goes to sleep mode and 
repeat the program.If there are no sensor readings are available, that error showed in the display unit. Flow chart under without wifi, as shown in Figure 4.

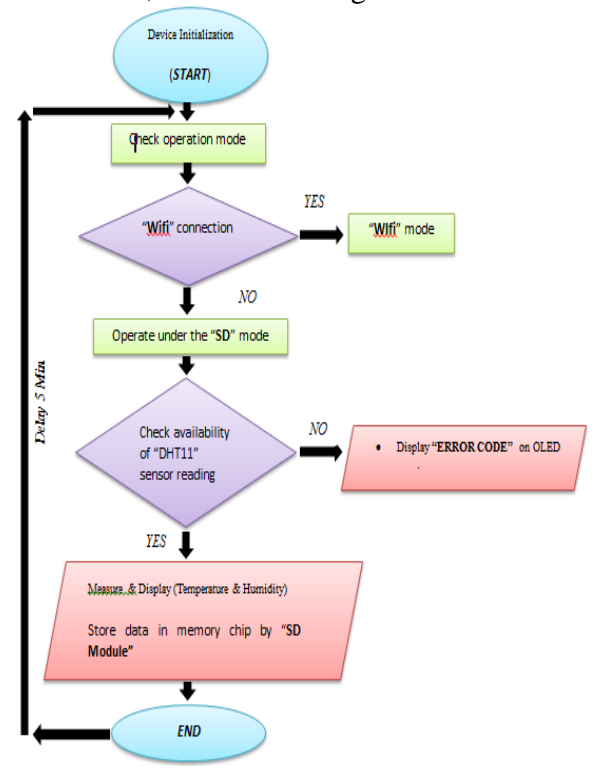

Fig 4 - Flowchart of Firmware \& code "Without Wi-Fi Mode"

\subsection{Mounting of the Device.}

Obtain information on temperature and humidity, this device can be mounted indoors or outdoor environment, therefore; there are two methods of mounting this device. Indoor mounting:- When it comes to more fixtures, this device is designed to be easily placed on the table or any rough surface. al besides, another sub-part can be attaché to hold this device on a smooth surface like a glass. The sub-part and how to connect it with the main device, as Fig 5. Outdoor mounting: When considering the outdoor environment, it has a mostly rough and heap surface. Therefore, a flexible \& adjustable belt with a velcro strip is designed to be able to connect to this device. The flexible belt and how to use it with outdoor application.
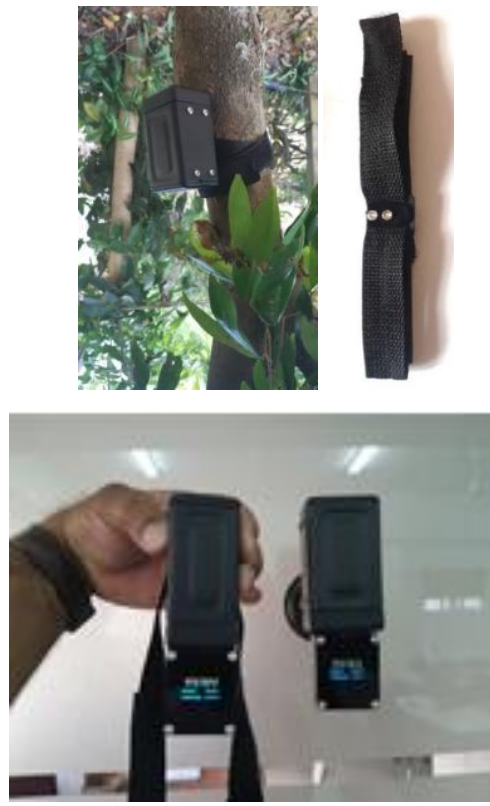

Fig 5 - Outdoor mounting

\subsection{Protected Body Design}

The protection body of the device is designed by SolidWorks software (Ex: Assembly, Simulation, check cross-section views)[14]. al, the $3 \mathrm{D}$ printing method is used to create the protection body parts by Acrylonitrile Butadiene Styrene (ABS) material [15].The final dimension of the device $40 \mathrm{~mm} \times 36 \mathrm{~mm} \times 80 \mathrm{~mm}$. There are 3 main components included in the protection body. Such as the top part of the body, the middle part of the body, the bottom part of the body. The top part of the protection body: This is the upper part of the protection body. The top wall is about $2 \mathrm{~mm}$ thick and the side walls are about $5 \mathrm{~mm}$. mainly in this part, there is a small opening hole in the upper wall which helps to remove the SD memory chip from the SD module. Simulation and real part respectively, as (see Figures $6 \& 7$ ).

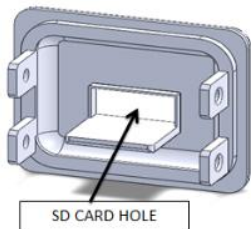

Fig 6- Simulation part

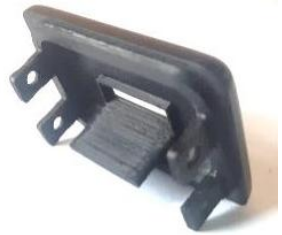

Fig 7 - Real part
The middle part of the body: This is the middle part of the protection body. This part helps to keep all the circuits. Such as PCB board ,NodeMCU board, DC-DC step-up booster, SD module and a Battery. When we considered cross-sectional views of the middle part is divided into two main chambers, one of which is built to store the battery and the other to store the PCB board with the related part. All the walls here are five-millimeter thicknesses. simulation and real part respectively, as (see Figures 8).

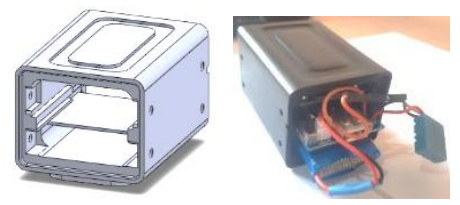

Fig 8 - 3D model and Real view part

Bottom part of the body: This is the bottom part of the protection body. That wall thickness is the same as the top part of the body. T we hole open to outside. One of those used to operate the main ON/OFF switch and others to hold the female port of the display unit. al, this part helps to retain the DHT11 sensor. Simulation and real part respectively, as (see Figures $9 \& 10$ ).

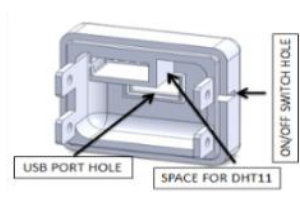

Fig 9-Simulation part
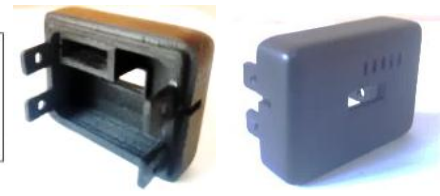

Fig 10- Real part

\subsection{Display Design}

The display unit of the main device was designed in SolidWorks and prototyped using a 3D printer. Bellow fig. 14 shows what are the main components of the display unit. When we consider the display design, we used 4 layers of films for better visualization. Display unit, as (see figure 11). 


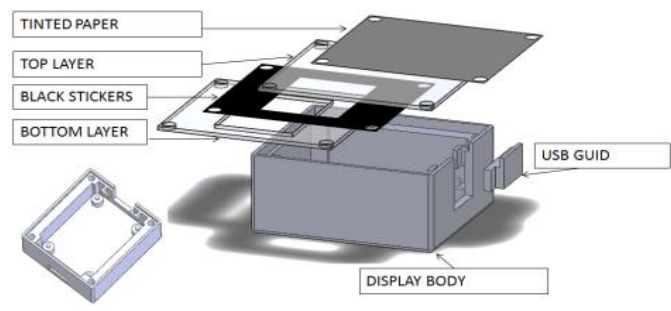

Fig 11 - Display design

\subsection{Method to Create Compact Device \& PCB Design.}

All this electrical component is connected to the PCB board[16] separately, a large amount of space $(150 \times 90) \mathrm{mm}$ is lost in our design, as (see Figures $12 \& 13$ ). we used the space between the PCB board and NodeMCU board to installing to these electrical components (DC-DC step-up booster and SD module) in figure 15 . Due to that, we were able to make a lightweight and compact product $(66 \times 35) \mathrm{mm}$ with low cost. To create a compact device, we have modified some electrical components. (DC-DC step-up booster and DHT11)

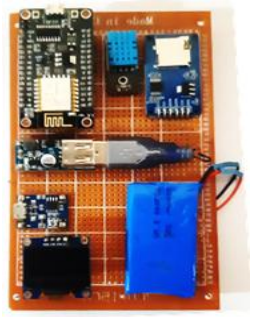

Fig 12 - Before the Compact

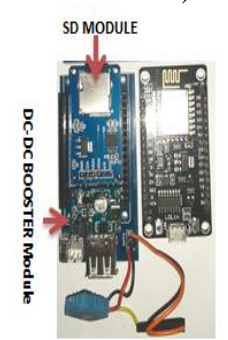

Fig 13- After Compact

\subsection{International Protection Rating (IP Code)}

The internal component of this device is covered with a protective body to minimize possible damage from the external environment., besides, it minimizes the potential damage from solids and liquids in the external environment under the following condition As defined in international standard EN 60529.First Digit(Solids): 5 (Protected against dust limited ingress, no harmful deposits.)Second Digit(Liquids): 1 (Protected against vertically falling drops of water, e.g. condensation. So the IP code of this device is IP51.3.0 International Protection Rating(IP CODE).

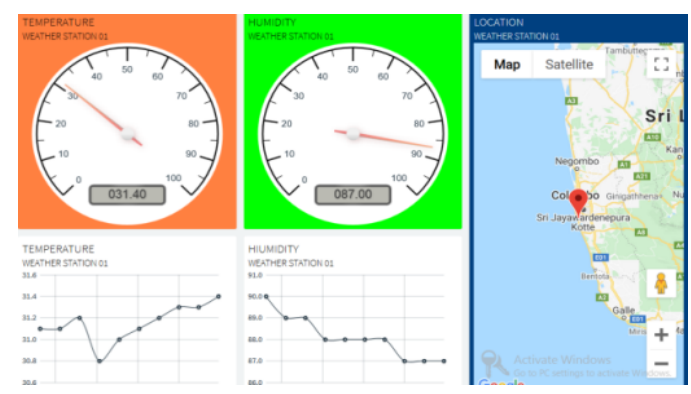

Fig 14 - Real Time monitoring

\section{EXPERIMENTAL RESULTS \\ 4.1 Data Monitoring Result}

In our experiment setup, we measured data (temperature \& humidity) continuously every five minutes throughout the week and stored that data in the data bucket of Thinger.io. Then that data (CSV data format) exported by E-mail. After that, we can be download data and analyzed it with MATLAB with smooth. Graphs of Temperature and humidity with time, as (See Figures 15).
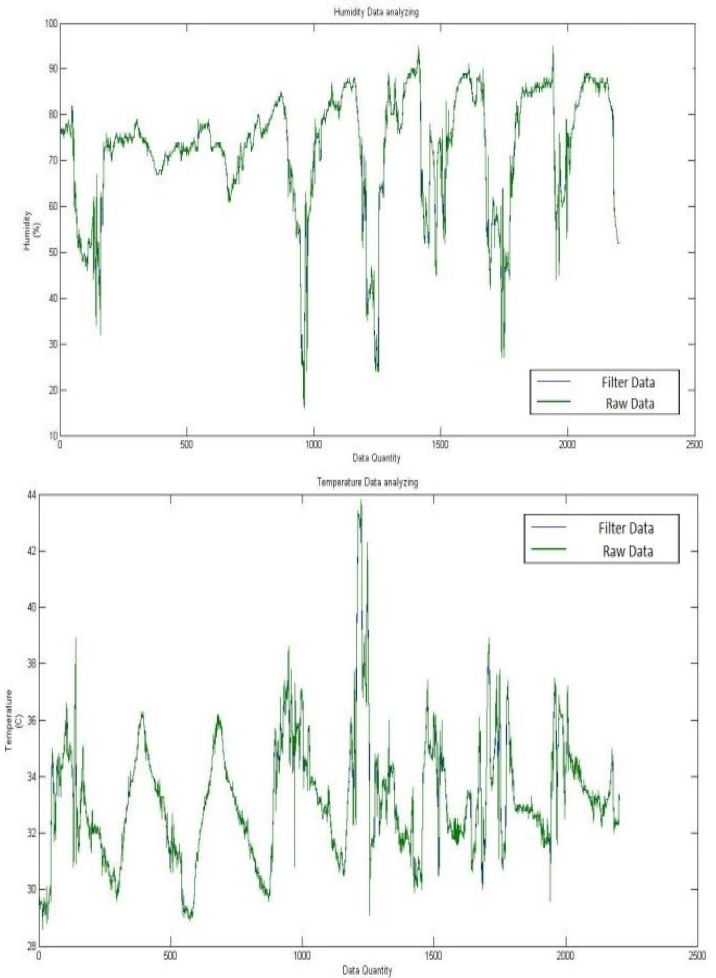

Fig 15 - Temperature with respectively Time

Note: These two graphs provide insight into the relationship between temperature and humidity, thereby that prove reducing the amount of water vapor that can be stored in a unit of volume as the temperature increases.

\section{CONCLUSIONS}

The paper describes the design of an IoT sensor node for monitoring humidity and temperature and how to design a smart device at a low cost. The average current consumption is measured by a power meter. The major challenge is to design a less power consumption devices. To solve this problem, we can be to used sleep mood function. (Device stay sleep mood at 4 minute and 55 seconds after that, it become operational condition at last 5 seconds)

Our other challenge is to develop a compact product. To meet those requirements, we have focused on three main factors. Such as :(1) Designed double-layer PCB. (2)Use the space between the NodeMCU board and PCB.(3)Using Solidworks software for simulation and assembly function. Although our device DHT11 sensor was calibrated using a calibrated thermometer. But in special cases, there are large fluctuations between the information provided by the sensor and real information given by the thermometer. T possible causes may contribute to these fluctuations, (1) Air convection around the sensor. (2) Dust particle deposition on the sensor. As a solution to these, we design a special protection body and cell cover with a small hole to install the DHT11 sensor. Another goal was to create an ergonomic product with a smooth surface. To achieve that goal, we are using the 3D printing method and laser cut. But in 3D printing, the outer surface is not smooth. So we had to wipe the surface with water 
sandpaper $(400,600,1200)$ to make a smooth surface. Also, a PCB coating was used to create this device to suitable for both internal and external environmental conditions (Excellent water repellency and corrosion prevention).

Finally, when a system error occurred, we needed to identify it. For this, we used the cloud base notification service.

\section{Calculation (With OLED display unit )}

Current consumption per 3 hours $(5 \mathrm{~V})=140 \mathrm{mAh}$

Current consumption per 1 hours $(5 \mathrm{~V})=140 / 3 \mathrm{mAh}$

Find current consumption $(3.7 \mathrm{~V})$

$$
I 1 \times V 1=I 2 \times V 2
$$

Where;

$\mathrm{I}_{1}=$ Current consumption per 1 hours at $(5 \mathrm{~V})$

$\mathrm{V}_{1}=5 \mathrm{~V}$

$\mathrm{I}_{2}=$ Current consumption per 1 hours at $(3.7 \mathrm{~V})$

$\mathrm{V}_{1}=3.7 \mathrm{~V}$

$$
\begin{gathered}
I 2=\left(\frac{I 1 \times V 1}{V 2}\right) \\
I 2=\left(\frac{46.6 m A \times 5 V}{3.7 V}\right)
\end{gathered}
$$

$\mathrm{I}_{2}=63.06 \mathrm{~mA}$

Find no of working hours

Battery Capacity $=700 \mathrm{mAh}$

Working hours $=(700 / 60.06)$

$$
=11.65 \mathrm{hrs}
$$

\section{REFERENCES}

[1] M. W. P.Maduranga, P. Saengudomlert, and HMN Dilum Bandara."Redundant Node Management in Wireless Sensor Networks with Multiple Sensor Types." 2018 National Information Technology Conference (NITC). IEEE, 2018.".

[2] A. V. S. N. Manoj and G. V. N. Deepak. K. Ladi, "'IOT based weather reporting system to find dynamic climatic parameters," 2017 International Conference on Energy, Communication, Data Analytics and Soft Computing (ICECDS), Chennai, 2017, pp. 2509-2513. ".

[3] Z. Yu, Z. Luo, and K. Wang. Z. Liu, "Development of Temperature and Humidity Acquisition Graphic User Interface," 2016 Sixth International Conference on Instrumentation \& Measurement, Computer, Communication and Control (IMCCC), Harbin, 2016, pp. 24-27.".

[4] M. W. P. Maduranga. Siththtikumar, "'Designing and Implementing an Arduino Based Low- Cost Automated Water Irrigation System for Home Gardens." International Research Symposium on Engineering Advancements SAITM, Malabe, Sri Lanka. 2016.".
[5] P. Kapoor and F. A. Barbhuiya., "'Cloud Based Weather Station using IoT Devices," TENCON 2019 - 2019 IEEE Region 10 Conference (TENCON), Kochi, India, 2019, pp.".

[6] V. Salehi and F. Abraham. J. Taha, "'Development of a Low Powered Wireless IoT Sensor Network based on MBSE," 2018 IEEE International Systems Engineering Symposium (ISSE), Rome, 2018, pp. 1-8. ".

[7] D. Kauling and Q. H. Mahmoud., "'Sensorian Hub: An IFTTT-based platform for collecting and processing sensor data," 2017 14th IEEE Annual Consumer Communications \& Networking Conference (CCNC), Las Vegas, NV, 2017, pp. 504-509".

[8] L. Yu, "'Matlab Programming Environment Based on Web," 2018 IEEE 4th Information Technology and Mechatronics Engineering Conference (ITOEC), Chongqing, China, 2018, pp. 509-512. ".

[9] Maduranga, M. W. P., and Ruvan Abeysekera. "IoT Based Monitoring and Intelligent Controlling for Plant Cultivation: A Case Study On Sandalwood Plant." Annual Conference - IET- Sri Lanka Network,2020

[10] Weerasinghe, YS P., M. W. P. Maduranga, and M. B. Dissanayake. "RSSI and Feed Forward Neural Network (FFNN) Based Indoor Localization in WSN." 2019 National Information Technology Conference (NITC). IEEE, 2019.

[11] P. Suryaganesh, M. Abishek and U. Benny, F. Margret Sharmila, "'Iot Based Smart Window using Sensor Dht11," 2019 5th International Conference on Advanced Computing \& Communication Systems (ICACCS), Coimbatore, India, 2019, pp. 782-784.".

[12] A. F. R. Leal and B. R. P. da Rocha, ""A low-cost lightning data logger using GPS time and SD card memory to the Amazon region," 2015 International Symposium on Lightning Protection (XIII SIPDA), Balneario Camboriu, 2015, pp. 318-321. ".

[13] J. Yoo et al., "'Highly Flexible AM-OLED Display With Integrated Gate Driver Using Amorphous Silicon TFT on Ultrathin Metal Foil," in Journal of Display Technology, vol. 6 , no. 11 , pp. $565-570$, Nov. 2010. ".

[14] X. Jing and W. Xiaoyan. P. Yuqing, "'Research and Realization on Architectural 3D Model of Architectural Process Simulation System," 2009 Second International Workshop on Computer Science and Engineering, Qingdao, 2009, pp. 543-547.".

[15] K. Chung, H. Yu and S. O. Yang S. Kim, "'G-code conversion from 3D model data for 3D printers on Hadoop systems," 2017 4th International Conference on Computer Applications and Information Processing Technology (CAIPT), Kuta Bali, 2017, pp. 1-4.".

[16] Tae-Heon Lee, Sang-Kook Kim, Jin-Yong Jeon and JaeKyung Wee Chang-Gyun Kim, "'Module design using 2layers PCB for an automotive BLDC motor driver," 2008 Electrical Design of Advanced Packaging and Systems Symposium, Seoul, 2008, pp. 109-112.". 